\title{
Elk and deer diets in a coastal prairie-scrub Mosaic, California
}

\author{
PETER J. P. GOGAN AND REGINALD H. BARRETT
}

\begin{abstract}
Atthors are animal ecologist, Greater Yellowstone Field Station, Midcontinent Ecological Science Center, National Biological Service, P. O. Box 168, Yellowstone National Park, Wyoming 82190; and professor, Department of Enviromental Science, Policy and Management, University of California, Berkeley 94720 . At the time of the research, the senior author was research associate, Department of Forestry and Resource Management, University of California, Berkelev, California.
\end{abstract}

\begin{abstract}
We examined the diets of reintroduced tule elk (Cervus elaphus nannodes Merriam) and resident Columbian black-tailed deer (Odocoileus hemionus columbianus Richardson) inhabiting the coastal prairie-scrub mosaic of Tomales Point, the northernmost portion of the Point Reyes Peninsula, Calif., during 1979-81. The elk diet differed between years whereas the deer diet did not. The pattern of seasonal quality of elk and deer diets, as measured by fecal nitrogen (FN) was similar between species and years. This was achieved although botanical composition differed between herbivores in some seasons. Dietary overlap was lowest in the wet winter months when fecal nitrogen was highest and vegetative standing crop was lowest. Conversely, dietary overlap was highest in the dry summer months when fecal nitrogen was lowest and vegetative standing crop highest. Both herbivore species showed selection and avoidance of certain plant species in June of both years. These findings are compared to other cervid-habitat systems.
\end{abstract}

Key Words: Tule elk, Cervis elaphus nannodes, Columbian black-tailed deer, Odocoileus hemionus columbianus, food habits, dietary overlap, dietary quality, coastal prairie-scrub mosaic, California

Tule elk (Cervis elaphus nannodes Merriam) and Columbian black-tailed (Odocoileus hemionus columbianus Richardson) deer were sympatric throughout much of central-coastal California including the Point Reyes Peninsula in prehistoric times. Tule elk were extirpated from most of their range by the $1860 \mathrm{~s}$

Research was funded by the National Purk Service under Contract CX 800-90029 and Califormia Agriculture Experiment Station Project 3501-MS. The senior author was a Rob and Bessie Welder Wildlife Foundution Fellow and this manuscript is Welder Wildlife Foundation Contribution 435. Authors thank C. F. Truman for carrying out the microhistological analy'ses of fecal samples with assistance from J. Dushek in preparation and identufication of reference material. $R$. C. Clurk and K. T. Lathrop completed the vegetation sampling. L. M. Merkle and E. Korpi typed drafts of the manuscript. K. J. Jenkins kindly reviewed an earlier draft of this manuscript. S. Broadbent provided editorial review.

Manuscript accepted 7 Nov. 1994.
(McCullough 1969). Deer densities have varied since that time in response to market hunting, habitat modification, and introduction of exotic plants and domestic stock. They are believed to be higher now than in pristine times (Longhurst et al. 1952, 1976). Since the mid-1970s, joint federal and state efforts to increase tule elk numbers by reintroductions to areas within the original range have resulted in these sympatric species sharing historically modified habitats throughout portions of their prehistoric ranges (Fowler 1985, Clark 1986). The 1978 reintroduction of tule elk and 1979 removal of livestock from a coastal prairie-scrub mosaic on the Point Reyes Peninsula provided an opportunity to examine cervid-range interactions while tule elk were at low numbers. However, we judged the nutritional condition of the reintroduced elk to be poor as indicated by copper deficiency and adult mortality (Gogan et al. 1989), antler anomalies (Gogan et al. 1988), and a zero growth rate for the herd between 1979 and 1981 (Gogan and Barrett 1986). Such characteristics are more descriptive of an elk herd utilizing limited forage resources rather than one where forage resources are abundant. Hence, we hypothesize that competative constraints between elk and deer were pronounced throughout the life of this study.

Interspecific competition is an important factor in shaping both niche dimensions and the geographic distribution of species (Wiens 1979). The role of dietary overlap as a measure of the degree of competition between sympatric species remains unclear (Martin 1993). Dietary selection has been considered an ecosystem process directly influencing community structure and interactions within the biotic community (Ellis et al. 1976). Limited resources may decrease foraging options and thereby promote increased interspecific or intraspecific competition. Alternately, high dietary overlap may reflect a lack of competition which permits common utilization of resources (Strong 1983). Putman (1986) found that dietary overlap among 5 sympatric species of large grazing herbivores was greater than overlap in habitat use or activity pattern but that overlap indices were reduced when all factors were considered rather than diet alone. We examine our findings relative to the hypotheses that 1) dietary overlap is least at times of low forage abundance and greatest at times of high forage abundance (Hobbs et al. 1983, Schoener 1986) or 2) that overlap decreases as forage biomass increases and, conversely, 
overlap increases as forage biomass decreases (Westoby 1974, Nudds 1980).

\section{Study Area}

The study was undertaken on the Point Reyes Peninsula $65 \mathrm{~km}$ NW of San Francisco, Calif. A 2.5-m drift fence limits tule elk to approximately 1,030 ha of Tomales Point, a ridge surrounded by salt water at the northern end of the Point Reyes Peninsula. The north-south oriented ridge ranges in elevation from 0 to $160 \mathrm{~m}$, sloping westward toward the Pacific Ocean and eastward toward Tomales Bay. East-west oriented valleys open from the east side of the ridge to Tomales Bay at sea level.

Tomales Point supports a species-rich mosaic of coastal prairie and coastal scrub common to much of the coastline of northern California (Barbour et al. 1973, Kuchler 1977). Many exotic plant species have become established in the past 200 years (Heady et al. 1977). Coastal prairie on Tomales Point is characterized by exotic grasses such as silver hairgrass (Aira caryophyllea L.), soft chess (Bromus mollis L.), ripgut brome (B. rigidus Roth), brome fescue (Festuca dertonensis All.), annual ryegrass (Lolium multiflorum Lam.), and perennial ryegrass (L. perenne L.) along with the native grasses meadow barley (Hordeum brachyantherum Nevskii) and purple needlegrass (Stipa pulchra Hitchc.) (Gogan 1986). Common forbs include the native perennial cow parsnip (Heracleum lanatum Michx.) and exotic English plantain (Plantago lanceolata $\mathrm{L}$.). The coastal prairie is interspersed with stands of coastal scrub characterized by bush lupine (Lupinus arboreus Sims.) and coyote bush (Baccharis pilularis DC.). Miner's lettuce (Montia perfoliata Donn) is common beneath the bush lupine.

Dense stands of coastal scrub on the eastern side of Tomales Point are characterized by sparse occurrence of grasses and forbs except cow parsnip and the presence of woody species such as poison oak (Rius diversiloba T. \& G.) and wax-myrtle (Myrica californica Cham. \& Schlecht.). Coast sagebrush (Artemisia pycnocephala DC.) is limited to south-facing slopes. Sandbar willow (Salix hindsiana Benth.) occurs adjacent to springs and seeps, particularly at the mouths of streams flowing into Tomales Bay. Stands of Monterey cypress (Cupressus sargentii Jeps.) and eucalyptus (Eucalyptus spp.. L'Herr.) were planted at 2 ranch complexes in the latter 1800s and mid-1930s, respectively. More than 290 plant species have been recorded on Tomales Point (Lathrop and Gogan 1985).

The Mediterranean-type climate of the central California coast is characterized by year-round moderate temperatures, summer fog and northwesterly winds, and fall and winter rains. Mean daily temperatures average $11.4^{\circ} \mathrm{C}$ and vary from $12.1^{\circ} \mathrm{C}$ in July to $0.0^{\circ} \mathrm{C}$ in January, although winter storm patterns are often interspersed with periods of 1 to 2 weeks of temperatures above $15^{\circ} \mathrm{C}$ and calm winds (U.S. Department of Commerce 1942, Howell 1970). Mean annual rainfall for 1926-83 at Inverness on the Point Reyes Peninsula is $952 \mathrm{~mm}$ (Gogan 1986). Advective fogs from May through August provide additional moisture. Shrubs at Tomales Point may be capable of collecting fog drip comparable to the $304 \mathrm{~mm}$ gathered by fog intercept screens in open grasslands $22 \mathrm{~km}$ from the coast in Humboldt Co., Calif. (Azevedo and Morgan 1974). Hence, total precipitation at Tomales Point may be considerably higher than indicated by rainfall records alone.
Plant phenology is linked to precipitation patterns with herbs and grasses beginning to tiller following the onset of the fall rains and beginning to flower in early spring. Most herbaceous vegetation is dry by mid-August, with the exception of English plantain.

A drought occurred in California in 1976 and 1977 with precipitation at Inverness approximating $33 \%$ and $66 \%$, respectively, of the 1926-83 average (Gogan 1986). Precipitation was at or above average from 1978 through 1981 (Gogan 1986). Range conditions were judged to be in fair to good condition in winter 1978 based upon plant species composition and forage value and percent cover (Dougherty et al. 1978), although they deteriorated during the remainder of that year (Gogan 1986). Range conditions, as judged by species diversity and standing crop, improved during this study (Gogan 1986).

Ten adult tule elk were translocated from Merced County, Calif., to Tomales Point in March 1978. The 2 adult male and 8 adult female elk were acclimated in a 1.2-ha holding pen and provided alfalfa hay as a dictary supplement until September 1978 when the adult elk and 7 calves born in the holding pen were released to become free-ranging on the northern most 1,030-ha of Tomales Point. Barbed wire livestock fences limited the movements of the newly released elk within this area (Ray 1981) until they were dismantled in November 1979. Elk movement south is prevented by a 2.5 -m drift fence. By the spring of 1979 , the elk exhibited signs of nutritional stress. Two adults died of copper deficiency in July 1979 (Gogan et al. 1989). Both adult male elk exhibited antler anomalies (Gogan et al. 1988). The remaining elk consumed the approximately $16 \mathrm{~kg} /$ day (wet weight) of fresh alfalfa, alfalfa feed, and commercial livestock feed supplements provided daily between mid-September 1979 and early February 1980 ( $1 \mathrm{~kg} /$ animal/day) and on alternate days thereafter through April 1980 ( $0.5 \mathrm{~kg} / \mathrm{animal} /$ day $)$, when they ceased taking it. Only a few deer took the supplements. The rate of population growth for the reintroduced elk for 1978-1981, coincident with this study, was 0 , but approximated the maximum potential from 1981 to 1984 (Gogan and Barrett 1986). Population size has continued to increase through 1994 (W. Shook, pers. comm.). Thus, we consider the range conditions to be sufficiently poor throughout this study to characterize the elk herd as comparable to one at ecological carrying capacity.

The released elk used only the southern half of the study area from September through December 1978 (Ray 1981), prior to provision of dietary supplements. They continued to use only the southern portion of the study area from July 1979 through September 1981, or for some 17 months after cessation of supplemental feeding (Gogan 1986). Our observations of elk at Point Reyes from 1982 through 1986 indicate that the elk used both the southern and northern portions of the study area subsequent to this study. Beef cattle were stocked at $0.4 /$ ha until November 1979, when they were removed. Resident black-tailed deer occurred at a relatively high density of $0.3 /$ ha (Gogan 1986).

\section{Methods}

\section{Diets and Dietary Quality}

Diets of elk and deer were determined by microhistological identification of plant epidermal fragments in fecal material (Sparks and Malechek 1968, Vavra and Holechek 1980). A fresh composite fecal sample ( $\geq 10$ pellets from $\geq 10$ pellet groups) was collected in the middle of each month by observing animals until 
they defecated for the 13 to 17 elk from October 1979 through September 1981. Composite fecal samples for deer $(\geq 10$ pellets from $\geq 15$ pellet groups) were collected from the ground in the middle of each month from the southern half of Tomales Point from December 1979 through September 1981.

Fecal samples were frozen until analyzed, at which time they were oven-dried and ground through a 1 -mm screen. Each sample was bleached until white (2-4.5 hours) and stained with hematoxylin and safranine prior to mounting on microscope slides in a gum arabic-Karo syrup medium (Truman el al. 1983). Twentyfive fixed fields on each of 5 slides were examined through a compound microscope at $100 \mathrm{x}$. Frequency of each plant species was converted to an estimate of percent composition by dry weight (Hansen et al. 1977). Portions of the pooled and ground monthly fecal samples from elk and deer used to determine food habits were analyzed for nitrogen content by the Kjeldahl method (Horowitz 1980).

\section{Dietary Overlap}

Dietary overlap was measured for elk and deer at the plant species level by use of a simple overlap index (Hurlbert 1978, Schoener 1986), i.e., the sum of the proportions of each plant species common to both species' diets (Schoener 1970). The index may vary from 0 to $100 \%$. This index was recommended by Abrams (1980) and has been used in various studies of dietary overlap in sympatric large herbivores (Anthony and Smith 1974, Leslic et al. 1984, Elliott and Barrett 1985),

\section{Forage Abundance and Preference}

A $1-\mathrm{km}^{2}$ universal transverse mercator (UTM) grid of Tomales Point was further subdivided into 4-ha cells. Each cell was classified to 1 of 4 vegetative cover types: open grassland, lupine grassland, Bacharris grassland, and thick scrub. Plant species availability was determined in 18 cells randomly selected from a total of some 300 (Gogan 1986). One transect oriented east-west and another north-south were centered on each cell. One hundred intercept sampling points along each transect were determined using the step-point method (Evans and Love 1957); points were located 1 pace (ca. $1 \mathrm{~m}$ ) apart. All plant taxa contacting the pin were recorded. Sampling was limited to June to optimize the ability to identify plant species as the greatest number of forbs and grasses flower in mid-June (Barbour et al. 1973). A weighted botanical composition of the range was calculated as the product of the percentage of all 4-ha cells assigned to each vegetative cover type and the mean occurrence of a plant species in the subsample of the 18, 4-ha cells in that cover type. In some instances, plant taxa could not be identificd beyond genus; thus, the number of taxa was a minimum.

Preference was assessed for all plant types and for all plant taxa contributing $\geq 5 \%$ to the weighted botanical composition of the range or the diet of either herbivore species during June 1980 and 1981. Preference for specific dietary items was quantified by a linear food selection index (Strauss 1979). The index ranged from -1 to +1 ; positive values suggested preference and negative values suggested avoidance or inaccessibility.

Availability of herbaceous biomass was determined at the same $1 S$ sites sampled for species composition. The aerial portion of all herbaceous vegetation was clipped within a $0.093-\mathrm{m}^{2}$ wire frame using hand clippers with blades held flush to the ground. Two or 3 samples were clipped at each site. The biomass of other forage classes was not measured. Sample periods approximated the end of the wet season (February), early dry season (June), and late dry season (October). The clipped material was dried to a constant weight.

\section{Results}

\section{Diets and Dietary Quality}

A minimum of 24 plant species, of which $50 \%$ were grasses, each made up $\geq 5 \%$ of the elk diet in $\geq 2$ sample periods (Table 1). The dietary supplements of alfalfa and pelleted livestock feed provided to elk between mid-October 1979 and mid-April 1980 were not identified in the diet. Shrubs were eaten more in the fall and winter of 1979-80 than at any other time (Fig. 1). Elk fed more on grasses in fall-winter and forbs in the spring-summer of $1980-81$ than 1979-80. The majority of forbs eaten in both years was English plantain and miner's lettuce, with the former consumed predominantly from March to September and the latter for the relatively short period of March through May in both years (Table 1). Coast sagebrush was consumed in the winter months of 1979-80, but not at other times. Bush lupine was the only other shrub consumed by elk (Table 1). Sandbar willow was used in the drier months of June-October of both years, and Monterey cypress was used in November 1979 (Table 1). Grass intake increased from October to December 1979, and again from October 1980 through March 1981. There was a rapid transition from grasses to forbs between March and April 1981 (Fig. 1). There was a distinct difference between years in the elk diet; shrubs were common in the winter 1979-80 diet and virtually absent from the winter 1980-81 diet.

A minimum of 25 plant species each made up $\geq 5 \%$ of the deer diet in $\geq 2$ or more sample periods (Table 1). Use of forbs declined from a peak in the spring to a low in the autumn with a concurrent increase in shrub use that peaked in autumn (Fig. 1). English plantain was consumed from March to September and miner's lettuce from March through May in 1980 and 1981 (Table 1). Yarrow (Achillea borealis Bong.) was consumed in all months with peaks in February of both years and a second peak in August-September of 1981, but sorrel and dock (Rumex spp. L.) were only eaten in June-July of both years (Table 1). Thistles (Cirsium spp. Mill.) were eaten only in 1980. Among shrubs. bush lupine was taken predominantly from August 1980 through February 1981. Other shrubs such as poison oak were consumed sporadically. Three tree species were eaten in the autumn and winter. There was no obvious between-year variation in the deer diet.

Elk and deer both exhibited parallel seasonal patterns of fecal nitrogen with a peak in the wetter months of January through April, declining during the drier months of May through December in both years (Fig. 2). Elk fecal nitrogen was lowest in October 1979.

\section{Dietary Overlap}

Elk and deer diets were most similar in December 1979 , August and October 1980, March 1981, and May through July 1981; dietary overlap was highest in May 1981 (Fig. 3). Overlap was least in February 1980 and November 1980 through February 1981. Dietary overlap was greatest when elk and deer simultaneously consumed forbs, primarily English plantain, sorrel and 
Table 1. Recent composition of taxa making up $\geq 5 \%$ of elk diet in any 2 sample periods. The elk diet was sampled monthly from October 1979 through March 1980 and bimonthly from April 1980 through September 1981.

\begin{tabular}{|c|c|c|c|c|c|c|c|c|c|c|c|c|c|c|c|c|c|c|c|c|c|c|c|c|c|}
\hline Taxa & Year & & ct. & $\mathbf{N}$ & ov. & & ec. & & n. & $\mathrm{Fe}$ & b. & & Tar. & & $\mathrm{pr}$. & & lay & & in. & $\sqrt{J}$ & ul. & & ug. & & ep. \\
\hline & & Elk & Deer & Elk & Deer & Elk & Deer & Elk & Deer & $\overline{\text { Elk }}$ & Deer & $\overline{\mathrm{EIk}}$ & Deer & Elk & Deer & Elk & Deer & Elk & Deer & Elk & Deer & Elk & Deer & $\overline{\text { Elk }}$ & Deer \\
\hline $\mathrm{ORBS}$ & & & & & & & & & & & & & & & & & & & & & & & & & \\
\hline hilla & $1^{a}$ & 0 & - & tr & - & 0 & 2 & $1 r^{c}$ & - & 13 & 13 & 2 & tr & ir & 4 & - & 0 & 0 & 2 & 0 & tr & tr & 2 & 0 & 5 \\
\hline borealis & $2^{\mathbf{b}}$ & ir & 6 & 0 & 0 & 2 & 2 & 0 & tr & tr & 36 & 0 & 2 & 0 & 3 & 2 & 2 & 3 & ir & 2 & 8 & 2 & 28 & 2 & 10 \\
\hline Erodium & $\overline{1}$ & 0 & - & 0 & - & 0 & 0 & 3 & - & 0 & 2 & tr & 3 & 8 & tr & - & tr & 11 & 0 & 4 & tr & tr & 0 & tr & 0 \\
\hline spp. & 2 & 0 & 0 & 0 & ir & 0 & 0 & 0 & 0 & 0 & tr & 2 & 0 & 3 & 3 & 6 & 4 & 6 & tr & tr & tr & 0 & 0 & 0 & 0 \\
\hline Iris & 1 & 0 & - & 0 & 一 & ir & tr & tr & - & 0 & 0 & 0 & 0 & 0 & 0 & - & 0 & 0 & 0 & 0 & 0 & 0 & 0 & 0 & 0 \\
\hline douglasiana & 2 & tr & 0 & 0 & 0 & 0 & 0 & 0 & 0 & 8 & 0 & tr & 0 & $\operatorname{tr}$ & 0 & 0 & 0 & tr & 0 & 0 & 0 & 0 & 0 & 0 & 0 \\
\hline Lobularia & I & 4 & - & tr & - & 0 & 0 & 0 & - & 0 & 0 & 0 & 0 & 0 & 0 & - & 0 & 0 & 0 & 0 & tr & 5 & 2 & 2 & 3 \\
\hline maritima & 2 & tr & 2 & 0 & 0 & 0 & 9 & 0 & $\mathrm{tr}$ & 0 & 0 & 0 & 0 & 0 & 0 & 0 & 0 & 0 & 0 & 4 & tr & 2 & tr & 4 & 0 \\
\hline Montia & 1 & 0 & - & 0 & - & 0 & 0 & 0 & - & 0 & 0 & 14 & 8 & 8 & 8 & - & 13 & 2 & Ir & 0 & tr & 0 & 0 & 0 & 0 \\
\hline perfoliata & 2 & 0 & 0 & 0 & 0 & 2 & 0 & 0 & 0 & 0 & 9 & 19 & 20 & 75 & 12 & 9 & 17 & 2 & 0 & 6 & 0 & 2 & 0 & 11 & 0 \\
\hline Plantago & 1 & 2 & - & $\operatorname{tr}$ & - & 0 & 0 & 5 & - & 0 & 0 & tr & 4 & 2 & 61 & - & 47 & 11 & 69 & 0 & 30 & 10 & 31 & 4 & 9 \\
\hline lanceolata & 2 & 3 & 2 & tr & 0 & 0 & 2 & $\pi$ & 3 & ir & 9 & 18 & 69 & 6 & 70 & 61 & 60 & 33 & 63 & 31 & 41 & 15 & 20 & 11 & 24 \\
\hline Potentilla & 1 & 0 & - & 0 & - & 0 & 0 & 0 & - & 0 & 2 & 0 & 8 & 0 & 0 & - & 0 & 0 & 0 & 0 & 0 & 0 & 0 & 0 & 26 \\
\hline spp. & 2 & 0 & tr & 0 & 0 & 0 & 0 & 0 & 0 & 0 & 0 & 0 & 0 & 0 & 0 & 0 & tr & 0 & 0 & 0 & 0 & 0 & 0 & 0 & 0 \\
\hline Rumer & 1 & 0 & - & 0 & - & 0 & 0 & 0 & - & 0 & 0 & tr & 5 & $\operatorname{tr}$ & $\operatorname{tr}$ & - & 2 & tr & 21 & tr & 6 & tr & 3 & 26 & 0 \\
\hline spp. & 2 & 3 & 0 & 2 & 0 & Ir & 0 & 0 & tr & 0 & 0 & 0 & tr & 4 & tr & 3 & 3 & 3 & tr & 10 & 16 & 7 & 4 & 0 & 0 \\
\hline Unknown & ] & 6 & - & 4 & - & 6 & tr & 13 & - & 0 & 3 & 3 & 2 & 7 & 2 & - & 7 & 6 & $\cdots$ & 16 & 13 & 20 & 18 & 4 & 3 \\
\hline Forbs & 2 & tr & 4 & tr & 2 & 2 & 8 & 6 & 8 & 0 & 0 & tr & tr & 0 & tr & tr & tr & 3 & ir & $\mathrm{tr}$ & tr & 0 & 2 & 4 & ir \\
\hline Total & 1 & 18 & - & 13 & - & 10 & 10 & 38 & - & tr & 25 & 32 & 47 & 43 & 88 & - & 76 & 57 & 94 & 40 & 72 & 47 & 56 & 37 & 56 \\
\hline Forb & 2 & 16 & 17 & 7 & 4 & 6 & 25 & 8 & 16 & 17 & 60 & 49 & 9.4 & 94 & 92 & 83 & 90 & 64 & 68 & 75 & 68 & 48 & 75 & 51 & 50 \\
\hline GRASSES/SEI & DGE & & & & & & & & & & & & & & & & & & & & & & & & \\
\hline Agrost & 1 & 0 & - & 0 & - & 3 & 0 & 0 & - & tr & 0 & 0 & it & 8 & 0 & - & 2 & 11 & 0 & 5 & 2 & 8 & tr & 13 & tr \\
\hline diege & 2 & 2 & 0 & 2 & 0 & 2 & 0 & 2 & tr & 0 & 0 & 0 & 0 & 0 & 0 & 0 & 0 & 0 & 0 & 2 & 0 & tr & 0 & 0 & 0 \\
\hline Aira & 1 & 0 & - & 0 & - & 5 & 0 & $\overline{0}$ & - & 0 & 0 & is & 0 & 0 & tr & - & 0 & 0 & $\mathrm{c}$ & 0 & & 0 & 0 & 0 & 0 \\
\hline cañophyllea & 2 & 2 & 0 & 0 & 0 & 0 & 0 & 0 & 0 & 0 & 0 & $\operatorname{tr}$ & tr & 0 & 0 & tr & 0 & 3 & 0 & 0 & & 5 & 0 & 0 & 0 \\
\hline Bromus & 1 & 0 & - & 0 & - & 0 & tr & 4 & - & 2 & 3 & 3 & 11 & 3 & tr & - & 2 & tr & U & $\mathrm{c}$ & & 0 & It & 0 & 0 \\
\hline spp. & 2 & 5 & $\operatorname{tr}$ & 0 & 0 & 0 & 0 & tr & tr & 5 & 9 & 7 & tr & 2 & ir & Ir & 0 & 2 & tr & 0 & c & 0 & 0 & 19 & 0 \\
\hline Damthonia & 1 & 0 & - & 0 & - & 0 & $\operatorname{tr}$ & 7 & 二 & tr & 0 & 2 & 5 & 0 & $\operatorname{tr}$ & - & 0 & 0 & 0 & 0 & 0 & 0 & 0 & 0 & 0 \\
\hline califonica & 2 & tr & ir & 0 & 0 & 0 & tr & 0 & tr & 0 & 0 & 0 & 0 & 0 & 0 & 0 & is & 0 & 0 & c & & 0 & 0 & 0 & 0 \\
\hline Festuca & 1 & 0 & - & 0 & - & 23 & 21 & 10 & - & 2 & 7 & 5 & 14 & tr & tr & - & $\operatorname{tr}$ & tr & c & c & & 0 & 0 & 0 & 0 \\
\hline dertonensis & 2 & 0 & 0 & 0 & 0 & 28 & 3 & 29 & $\operatorname{tr}$ & 15 & $\mathrm{tr}$ & 13 & 0 & 0 & 0 & 0 & 0 & 3 & c & c & ( & tr & $\pi$ & 0 & 0 \\
\hline$F_{\text {t'sluca }}$ & I & tr & - & 0 & - & 0 & 2 & 0 & - & 0 & 17 & 0 & 0 & 0 & tr & - & 0 & 0 & 0 & 0 & $\mathrm{c}$ & 0 & 0 & 0 & 0 \\
\hline rabra & 2 & 0 & 0 & tr & 0 & 9 & tr & 6 & 0 & 3 & 3 & 5 & 0 & 2 & $t s$ & 0 & 0 & 2 & 0 & 4 & c & 0 & 0 & 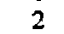 & tr \\
\hline Holcus & 1 & 0 & - & 0 & - & tr & 0 & 0 & - & 0 & 0 & ir & tr & 9 & tr & - & 0 & $\mathrm{tr}$ & 0 & 0 & c & 4 & 0 & - & 0 \\
\hline lanaturs & 2 & 23 & 0 & 41 & tr & 15 & 0 & 5 & tr & tr & 0 & tr & 0 & 0 & 0 & 0 & 0 & $\operatorname{tr}$ & 0 & 0 & & 15 & 0 & 16 & 0 \\
\hline Hordetm & 1 & Ir & - & 11 & - & 17 & 2 & 3 & - & 0 & 0 & 6 & 0 & tr & $\operatorname{tr}$ & - & $\operatorname{tr}$ & 0 & 0 & o & & 0 & 0 & . & 0 \\
\hline spp. & 2 & 3 & 0 & 0 & tr & 19 & 3 & 16 & tr & 5 & 2 & 4 & 0 & 0 & tr & 2 & tr & 0 & 0 & $\operatorname{tr}$ & c & 3 & 0 & 0 & 0 \\
\hline Jincus & 1 & 0 & 一 & 0 & - & 9 & 3 & 0 & - & 0 & 0 & 2 & 0 & 2 & tr & - & 0 & 0 & 0 & 0 & 0 & 6 & 0 & 0 & tr \\
\hline spp. & 2 & tr & 0 & 8 & 0 & tr & 0 & tr & 0 & 32 & tr & 5 & 0 & 0 & 0 & tr & 0 & $\pi$ & 0 & 0 & 0 & 2 & 0 & tr & 0 \\
\hline Lolitum & 1 & 0 & - & 0 & - & 6 & 0 & 6 & - & 2 & 0 & 6 & tr & 3 & 0 & - & 0 & $a$ & 0 & 0 & 0 & 2 & 0 & 14 & 0 \\
\hline perem & 2 & 0 & 0 & 6 & 0 & 2 & 0 & tr & 0 & 0 & $\mathbf{0}$ & 0 & 0 & 0 & 0 & 0 & 0 & 0 & 0 & 0 & 0 & 0 & 0 & 7 & 0 \\
\hline$P o a$ & 1 & 0 & - & 0 & - & 0 & 0 & 5 & - & tr & 0 & 2 & 0 & 5 & 0 & - & 0 & 0 & 0 & 0 & c & 0 & 0 & 0 & 0 \\
\hline$a n n t$ & 2 & 0 & 0 & 0 & 0 & 0 & 0 & 0 & 0 & 5 & 0 & 2 & 0 & tr & 0 & 0 & 0 & 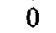 & 0 & c & & 0 & 0 & 0 & 0 \\
\hline Polypogon & 1 & 0 & - & 0 & - & 0 & 0 & 0 & - & 5 & 5 & 4 & tr & 0 & 0 & - & 0 & 0 & ( & $\mathrm{c}$ & & 0 & 0 & 0 & 0 \\
\hline monspeliensis & 2 & 0 & 0 & it & 0 & $\operatorname{tr}$ & 0 & 2 & tr & 0 & 2 & 2 & 0 & 0 & 0 & 0 & 0 & J & 0 & 0 & 0 & 0 & 0 & 0 & 0 \\
\hline Unknown & 1 & 0 & - & 22 & - & 1 & 3 & 0 & - & 5 & 0 & 5 & 2 & 13 & 2 & - & 5 & 28 & $\operatorname{tr}$ & 18 & & 12 & 6 & 15 & 4 \\
\hline gras & 2 & 9 & tr & 19 & $\operatorname{tr}$ & 13 & 3 & tr & 4 & 3 & 2 & 7 & tr & 0 & tr & 9 & 2 & 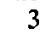 & 2 & & & 3 & 3 & 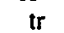 & 0 \\
\hline Total & 1 & 7 & - & 33 & - & 76 & 34 & 40 & - & 35 & $3 i$ & 41 & 41 & 56 & 7 & - & $1 \overline{4}$ & 41 & 2 & 23 & 4 & 32 & 8 & 50 & 5 \\
\hline grass & 2 & 46 & $\mathrm{tr}$ & 89 & $\operatorname{tr}$ & 91 & 10 & 91 & 9 & 77 & 20 & 50 & 2 & 6 & 2 & 16 & 4 & 31 & 2 & 15 & 4 & 30 & 4 & 45 & 2 \\
\hline SHR & & & & & & & & & & & & & & & & & & & & & & & & & \\
\hline Aremisia & 1 & 56 & - & 49 & - & 11 & 4 & 21 & - & 74 & to & 26 & 0 & 0 & $\mathrm{tr}$ & - & & 0 & & & & 0 & 4 & & 4 \\
\hline & 2 & 0 & 0 & 0 & 7 & 0 & 3 & 0 & 11 & 0 & $\mathrm{t}$ & 0 & & & 2 & 0 & & - & & & & IF & 7 & & 32 \\
\hline Bacch & 1 & 4 & - & $\operatorname{tr}$ & - & 0 & 6 & 0 & - & 0 & 0 & 0 & 0 & 0 & 0 & - & 0 & 0 & c & 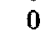 & ( & $\ddot{0}$ & 0 & 0 & 0 \\
\hline & 2 & tr & 0 & 0 & tr & 0 & 3 & 0 & 8 & 0 & tr & 0 & & 0 & 0 & 0 & 0 & & & & & 0 & 0 & & 0 \\
\hline Ceanothus & 1 & 0 & - & 0 & - & 0 & 0 & 0 & - & 0 & 0 & 0 & 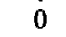 & 0 & 0 & - & 0 & 8 & ( & & & 0 & 0 & & 0 \\
\hline Ihrsiflo & 2 & 2 & 0 & 0 & 0 & 0 & 11 & 0 & 4 & 0 & tr & 0 & 0 & 0 & tr & 0 & 0 & 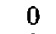 & 0 & 0 & & tr & 0 & 8 & 0 \\
\hline Lupmus & 1 & 10 & - & 0 & - & if & 9 & 0 & - & tr & 30 & 0 & 4 & & 3 & - & 2 & 0 & & & & 13 & 21 & & 24 \\
\hline sp & 2 & 32 & 56 & 2 & 29 & is & 2 & is & 31 & 0 & 11 & 0 & $\mathrm{tt}$ & 0 & 3 & 0 & 3 & 2 & 3 & ) & 0 & 4 & 7 & & 7 \\
\hline Lomicera & 1 & 0 & - & 0 & - & 0 & 12 & 0 & - & 0 & 0 & 0 & 0 & 0 & - & 0 & 0 & 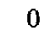 & 0 & tr & 0 & 0 & 0 & & 0 \\
\hline spp. & 2 & 0 & 0 & 0 & 0 & 3 & 0 & 0 & 0 & 0 & 5 & 0 & 0 & 0 & 0 & 0 & 0 & 3 & 10 & 5 & $\mathrm{c}$ & $\pi$ & 0 & & 0 \\
\hline Rhommus & 1 & 0 & - & 0 & 一 & 0 & 4 & 0 & - & 0 & 0 & 0 & . & 0 & 0 & - & 0 & 0 & 0 & 0 & 0 & 0 & 0 & 0 & 4 \\
\hline calif & 2 & 0 & 0 & 0 & $\operatorname{tr}$ & . & 0 & 0 & 7 & 0 & 0 & 0 & 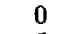 & 0 & 0 & 0 & 0 & 0 & & & & Ir & 0 & & 0 \\
\hline Rhus & $i$ & 0 & - & 0 & - & 0 & 0 & tr & - & 0 & 0 & 0 & 5 & 0 & 0 & 一 & 8 & 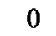 & 0 & 0 & 0 & 0 & 3 & 0 & 0 \\
\hline diven & 2 & 0 & 2 & 0 & 0 & 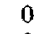 & 0 & 0 & 0 & 0 & 0 & 0 & 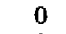 & 0 & 0 & 0 & 0 & 0 & & & 0 & 0 & 0 & & 0 \\
\hline Rubus & 1 & 0 & - & 0 & - & 0 & tr & 0 & - & 0 & 0 & 0 & 0 & 0 & tr & - & 0 & 0 & 0 & 0 & 0 & 0 & 0 & 0 & 0 \\
\hline 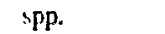 & 2 & 0 & 3 & 0 & 7 & 0 & 7 & 0 & 6 & 0 & 0 & 0 & 2 & 0 & 0 & 0 & tr & 0 & 0 & 0 & 20 & 3 & 0 & tr & 0 \\
\hline Unknown & 1 & 5 & - & 0 & - & 0 & 4 & 0 & - & 0 & 0 & 0 & -1 & 0 & 0 & - & 0 & 0 & 0 & 0 & 0 & 0 & 0 & 0 & 3 \\
\hline & 2 & 0 & 6 & 0 & 5 & 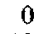 & 0 & 0 & 0 & 0 & 0 & 0 & 0 & 0 & 0 & 0 & 0 & 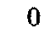 & 0 & 0 & 0 & 7 & 0 & & 0 \\
\hline Total & I & 76 & - & 50 & - & 13 & 46 & 22 & - & 74 & 33 & 26 & 9 & 0 & 5 & - & 11 & 0 & 2 & tr & 6 & 13 & 30 & 2 & 36 \\
\hline shrub & 2 & 35 & 76 & 2 & 86 & tr & 48 & $\operatorname{tr}$ & 67 & 0 & 19 & 0 & 3 & 0 & 6 & 0 & 6 & 5 & 27 & 5 & 25 & 17 & 16 & 3 & 43 \\
\hline
\end{tabular}

Table 1. Continued on page $333 .$. 
Table 1. Continued.

\begin{tabular}{|c|c|c|c|c|c|c|c|c|c|c|c|c|c|c|c|c|c|c|c|c|c|c|c|c|c|}
\hline \multirow[t]{2}{*}{ Taxa } & \multirow[t]{2}{*}{ Year } & \multicolumn{2}{|c|}{ Oct. } & \multicolumn{2}{|c|}{ Nov. } & \multicolumn{2}{|c|}{ Dec. } & \multicolumn{2}{|c|}{ Jan. } & \multicolumn{2}{|c|}{ Feb. } & \multicolumn{2}{|c|}{ Mar. } & \multicolumn{2}{|c|}{ Apr. } & \multicolumn{2}{|c|}{ May } & \multicolumn{2}{|c|}{ Jun. } & \multicolumn{2}{|c|}{ Jul. } & \multicolumn{2}{|c|}{ Aug. } & \multicolumn{2}{|c|}{ Sep. } \\
\hline & & Elk & Deer & Elk & Deer & Elk & Deer & Elk & Deer & Elk & Deer & Elk & Deer & Elk & Deer & Elk & $\overline{\text { Deer }}$ & Elk & Deer & Elk & Deer & Elk & Deer & Elk & Deer \\
\hline TREES & & & & & & & & & & & & & & & & & & & & & & & & & \\
\hline Cupressus & 1 & 0 & - & $\operatorname{tr}$ & - & 2 & 5 & 0 & - & 0 & 11 & 0 & 0 & 0 & 0 & - & 0 & 0 & 0 & 0 & 0 & 0 & 0 & 3 & 0 \\
\hline sargentii & 2 & $\mathrm{tr}$ & 0 & 2 & 0 & 0 & 10 & 0 & 0 & 0 & 0 & 0 & 0 & 0 & 0 & 0 & 0 & 0 & 0 & 0 & 0 & 0 & 0 & 0 & 0 \\
\hline Myrica & 1 & 0 & - & 0 & - & 0 & 3 & 0 & 一 & 0 & 0 & 0 & 4 & 0 & $\operatorname{tr}$ & - & 0 & 0 & 0 & tr & 0 & $\operatorname{tr}$ & 2 & 2 & 0 \\
\hline californica & 2 & 0 & $\operatorname{tr}$ & 0 & 8 & 0 & $\operatorname{tr}$ & 0 & 6 & 0 & 0 & 0 & 0 & 0 & 0 & 0 & 0 & 0 & 0 & 0 & $\operatorname{tr}$ & tr & 0 & 0 & 0 \\
\hline Salix & 1 & 0 & - & 0 & - & 0 & $\operatorname{tr}$ & 0 & - & 0 & 0 & 0 & 0 & 0 & 0 & - & 0 & $\operatorname{tr}$ & $\mathrm{tr}$ & 36 & 15 & 4 & 2 & 6 & 0 \\
\hline hindsiana & 2 & 2 & 2 & 0 & $\operatorname{tr}$ & 0 & 2 & 0 & 0 & 0 & 0 & 0 & 0 & 0 & 0 & $\mathrm{tr}$ & 0 & 0 & 3 & 3 & 2 & 2 & $\operatorname{tr}$ & $\operatorname{tr}$ & 2 \\
\hline Total & 1 & 0 & - & 2 & - & 2 & 9 & 0 & - & 0 & 11 & 0 & 4 & 0 & tr & - & 0 & $\mathrm{tr}$ & $\operatorname{tr}$ & 36 & 15 & 5 & 4 & 12 & $\operatorname{tr}$ \\
\hline tree & 2 & 3 & 4 & 2 & 8 & 0 & 15 & 0 & 6 & 5 & 0 & 0 & 0 & 0 & 0 & $\operatorname{tr}$ & 0 & 0 & 3 & 3 & 3 & $\operatorname{tr}$ & $\operatorname{tr}$ & $\operatorname{tr}$ & 2 \\
\hline
\end{tabular}

${ }^{\mathrm{a}}$ Year 1 = October 1979 - September 1980; ${ }^{\text {bYear } 2}=$ October 1980 - September $1981 ;{ }^{\mathrm{c}} \operatorname{tr}<2 \%$.

dock, and unknown forbs in 1980, and miner's lettuce and English plantain in 1981 (Table 1). Dietary overlap was minimal in February 1980 when elk ate chiefly grasses and coast sagebrush and deer ate forbs and bush lupine (Table 1). Another low in dietary overlap in September 1980 (Fig. 3) resulted from heavy use of dock and grasses by elk versus selection of shrubs and forbs other than dock by deer (Table 1). Dietary overlap remained low from November 1980 through February 1981 (Fig. 3) when
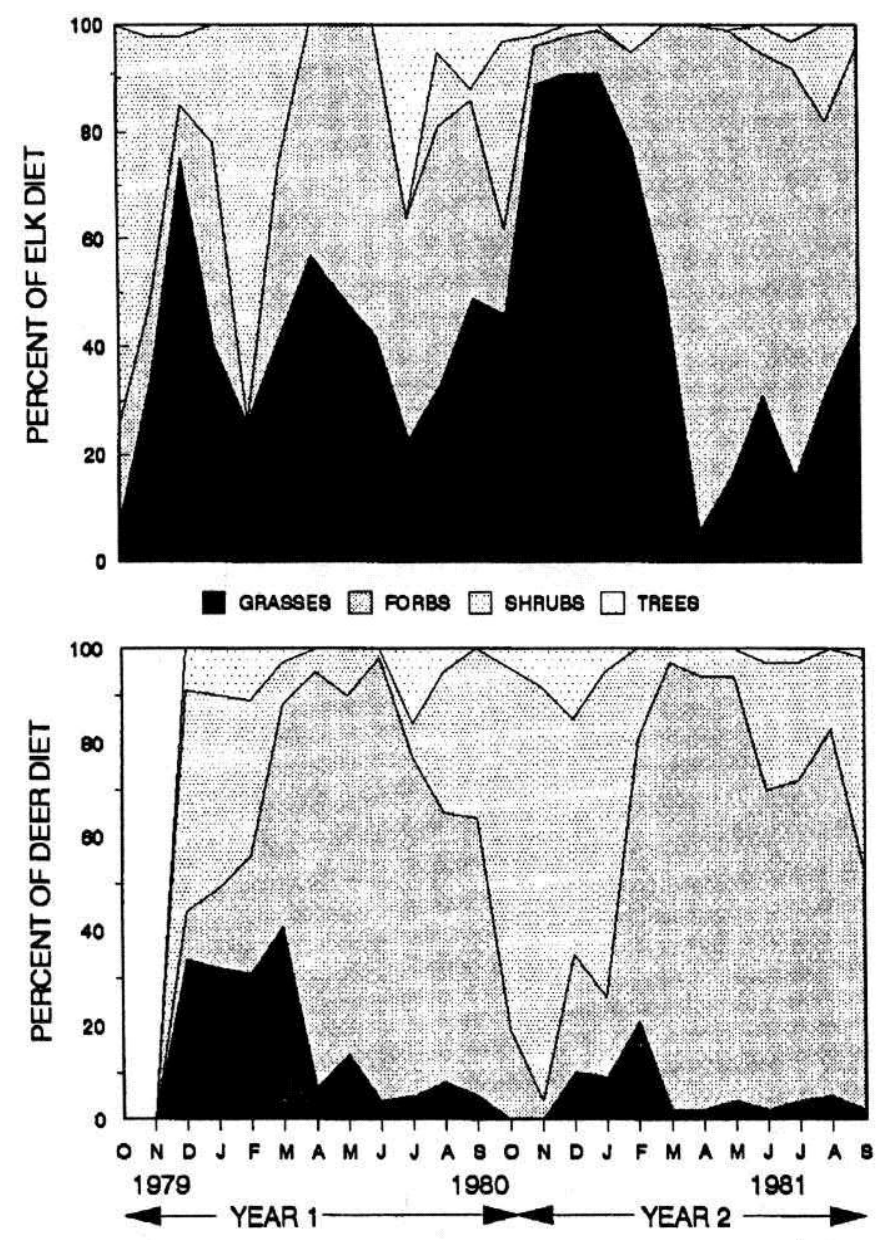

Fig. 1. Elk and deer use of forage classes at Tomales Point determined for elk by monthly samples from October 1979 through September 1981 and for deer by monthly samples from December 1979 through September 1981. the elk diet was $80-90 \%$ grasses and the deer diet $75 \%$ shrubs and forbs (Table 1).

\section{Forage Abundance and Preference}

Plant species were recorded at a total of 11,083 and 17,916 intercept points in June of 1980 and 1981, respectively. Grasses were the predominant plant cover. Forbs increased their portion of the plant cover from 1980 to 1981 . Herbaceous standing crop was at its lowest and highest in February and June, respectively, of each year; October 1980 was intermediate (Fig 4). Standing crop increased from 1980 to 1981 . Dietary selection was examined during June 1980 and 1981 when forbs predominated in the diets of both cervid species (Fig. 1). Only English plaintain was preferentially selected by either herbivore in June 1980 or 1981 (Table 2): Elk selected this forb only in 1981 whereas deer selected it in both years. Both herbivores avoided bromes (Bromus spp. L.) in both years and perennial ryegrass in 1980 .

\section{Discussion}

The value of fecal sample analysis to determine herbivore food habits has been scrutinized by a number of investigators. Holechek et al. (1982) concluded that fecal analysis tends to underestimate the proportion of forbs in the diet of a variety of ruminants, although some studies have reported this not to be the case (Todd and Hansen 1973, Anthony and Smith 1974, Kie et al. 1980). Leslie et al. (1983) reported seasonal variation in digestibility of plant species of the Olympic Peninsula, Washington, resulting in fecal analysis consistently underestimating the proportion of forbs and shrubs in the diet. We assumed bias in determining botanical composition of elk and deer diets at Tomales Point was consistent between herbivore species and tended to underestimate preference for forbs. This pattern may have contributed to the failure to detect in fecal samples the alfalfa and pelleted feed provided to elk from mid-September 1979 through April 1980 (Table 1). Another contributing factor may have been the small volume of supplements $(0.5 \mathrm{~kg} / \mathrm{animal} / \mathrm{day}$ wet weight) relative to total dietary intake $(4 \mathrm{~kg} / \mathrm{animal} / \mathrm{day}$ dry weight) (Nelson and Leege 1982). Biases in estimating range species composition with the step-point method are considered small when only direct contacts with the pin are recorded (Strauss and Neal 1983) as was done for this study (Gogan 1986). However, the number of large plants, in this study shrubs, may be overestimated (Strauss and Neal 1983). 
Table 2. Elk and deer preferences and standard errors (SE) for plant species making up $\geq 5 \%$ of either herbivore diet for June 1980 or 1981. Preference index is relative use minus relative availability (Strauss 1979); asterisks indicate preferences different $(P<0.10)$ from 0 .

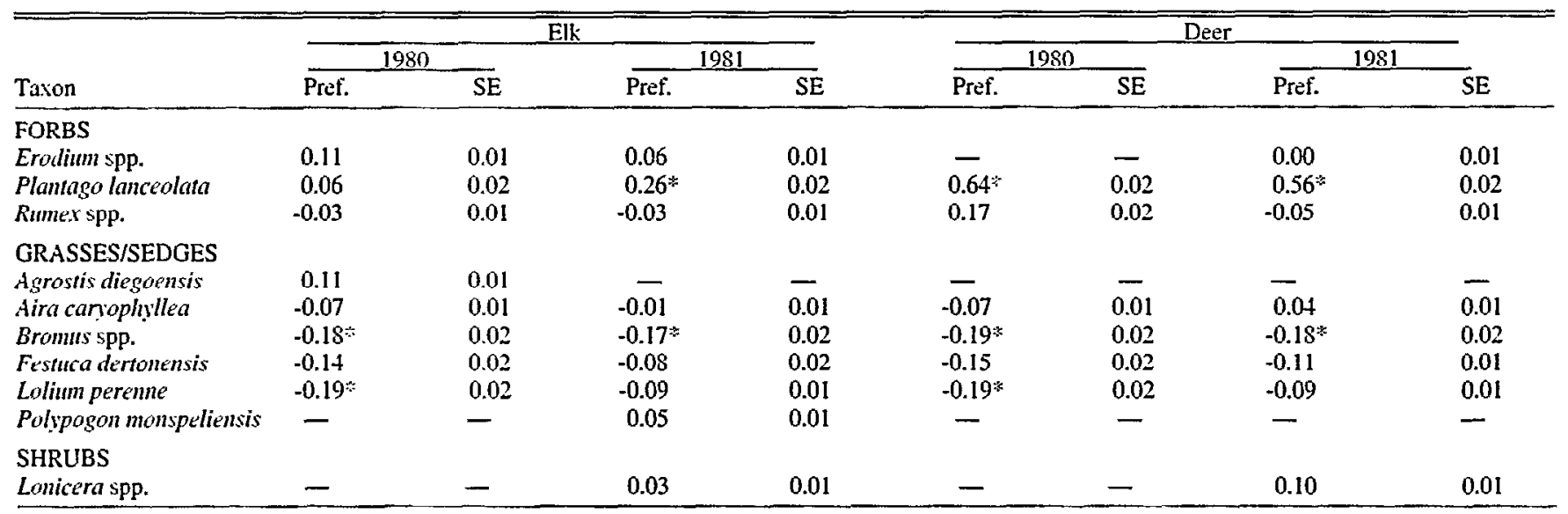

Tule elk at Tomales Point feed mainly on forbs and grasses. The high use of forbs by elk at this site is somewhat atypical as elk are generally considered grazers (Nelson and Leege 1982). Tule elk in the Owens Valley, Inyo County, Calif., however, also consumed forbs extensively in the spring and summer (McCullough 1969). Shrubs were a major component of the elk diet during the fall and winter of 1979-80, but less so during the same period of 1980-81. Willows were eaten only in the summer months. In contrast, tule elk in the Owens Valley fed on shrubs in the summer and willow in the winter (McCullough 1969). Elk at Tomales Point consumed grasses year-round, but primarily during winter. Grasses made up $>50 \%$ of the year-round diet of tule elk reintroduced to the Diablo Range. Santa Clara County, Calif., (Phillips 1985) and of Roosevelt elk at Prairie Creek Redwoods State Park, Humboldt County, Calif. (Harper et al. 1967). Roosevelt elk in forests on the Olympic Peninsula, Wash., showed pronounced seasonal shifts in the utilization of grasses, but consumed a maximum of only $26 \%$ in the fall (Leslie et al. 1984).

The high consumption of forbs and shrubs by black-tailed deer at Tomales Point is consistent with its classification as an intermediate feeder (Anderson and Wallmo 1984). Black-tailed deer on Tomales Point consumed far more shrubs (Fig. 1) than those immediately to the south where much of the coastal scrub has been cleared and seeded to grasses and forbs (Elliott and Barrett 1985). Similarities in dietary patterns between black-tailed deer in both areas include maximum consumption of grasses in the winter months and maximum consumption of forbs in the summer (Fig. 1, Elliott and Barrett 1985). Dietary patterns of deer in inland areas of central California are characterized by heavy reliance on browse throughout the year with some intake of forbs in the late winter and spring (Taber and Dasmann 1958,

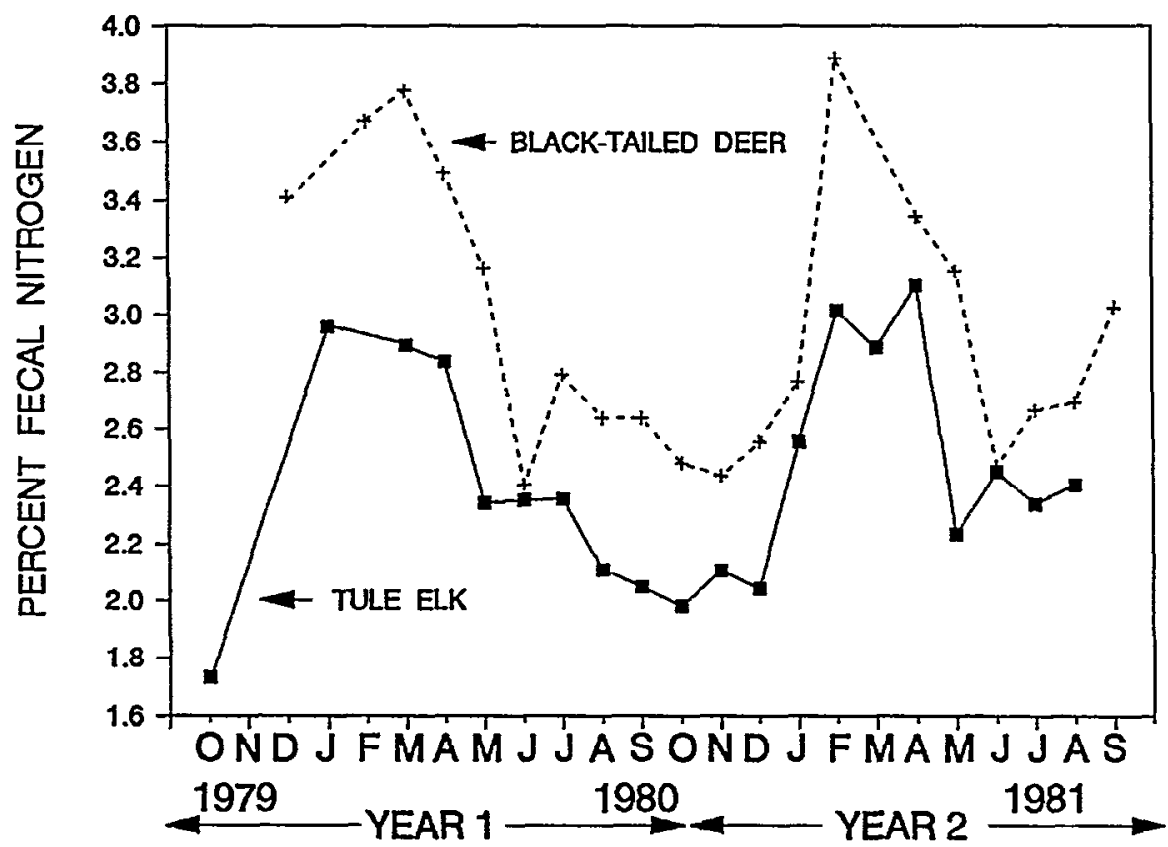

Fig. 2. Percent nitrogen content of elk and deer fecal samples determined for elk by monthly samples from October 1979 through September 1981 and for deer by monthly samples from December 1979 through September 1981 at Tomales Point. 


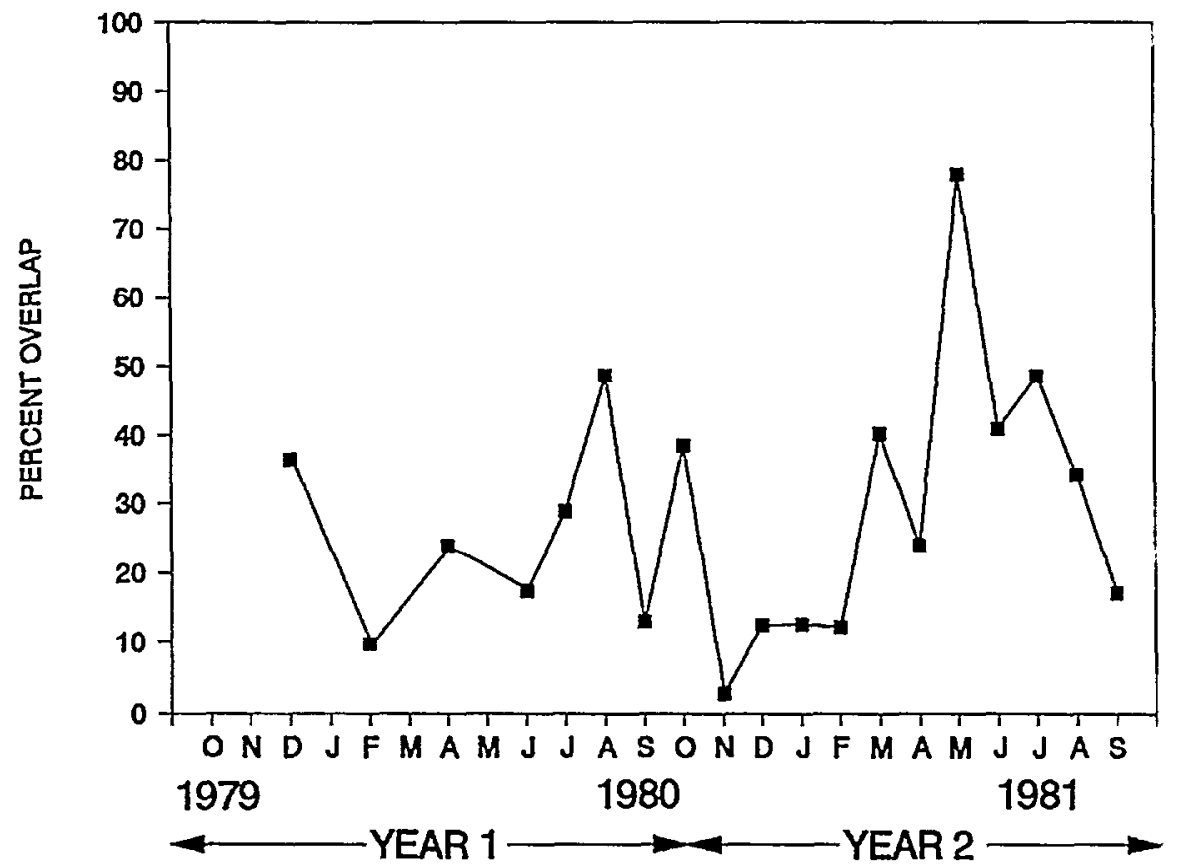

Fig. 3. Monthly dietary overlap of elk and deer from December 1979 through September 1981 at Tomales Point.

Longhurst et al. 1979). Similarly, black-tailed deer of the Olympic Peninsula, Wash., used browse extensively year-round but consumed forbs primarily in the summer months (Leslie et al. 1984).

Elk fecal nitrogen (FN) levels were consistently lower than those for deer with the exception that deer also had low levels in June of both years (Fig. 2). However, such differences between species need not reflect differences in dietary quality (Leslie and Starkey 1987) but may be modified by differences in rates of ingestion of plants containing tannins, such as shrubs or forbs (Hobbs 1987). The elk fecal nitrogen levels were consistent between years (Fig. 2) in spite of the between-year differences in dietary composition (Fig. 1). However, elk fecal nitrogen levels for winter 1980 may have been elevated by the high tannin levels found in shrubs (Hobbs 1987). An ability to maintain nutritional homeostacy by varying dietary intake has been reported previously for Rocky Mountain elk (C. e. nelsoni Bailey) (Hobbs et al. 1981). The general fecal nitrogen patterns for both elk and deer indicated dietary quality was highest in the winter wet season (Fig. 2). Similarly, fecal nitrogen levels for Roosevelt elk at Prairie Creek Redwoods State Park, Humboldt County, Calif., were highest in the winter months of January through April and lowest in the late summer and fall months of September through November (Gogan and Barrett 1994). In contrast, fecal nitrogen levels in Roosevelt elk and black-tailed deer on the Olympic Peninsula, Wash., were low in winter highest peak in the spring and summer. Such differences in fecal nitrogen levels likely reflect differences between peak forage production in the Mediterranean climate of coastal California relative to the temperate climate of the Olympic Peninsula. Similarly, fecal nitrogen levels of black-tailed deer in interior northern California were highest in the summer months when forage conditions peaked
(Kie and Burton 1984).

Low fecal nitrogen in tule elk at Tomales Point in October 1979 (Fig. 2) is below the minimal nutritional value for maintenance as calculated by Nelson and Leege (1982). It occurred concurrently with clinical signs of copper deficiency in the elk during the summer and fall of 1979 (Gogan et al. 1989), including antler anomalies in both adult males present at the time (Gogan et al. 1988). Comparable calculations for Roosevelt elk on the Olympic Peninsula, Wash., based upon fecal nitrogen levels reported by Leslie and Starkey (1985) indicate that these animals were more severely nutritionally stressed in 2 consecutive winters than were the tule elk at Tomales Point in October 1979. Yet no overt signs of nutritional stress were reported in Roosevelt elk.

We interpret differences in diets between elk and deer at Tomales Point, and positive and negative forage preference index values for both herbivores (Table 2), as indicative of dietary selection. The lowest dietary overlap between elk and deer occurred during the winter wet season when plants were relatively nutritious as indicated by fecal nitrogen levels (Fig. 2), but standing crop biomass was low (Fig. 4). The greatest dietary overlap occurred in the summer dry season when fecal nitrogen levels were low, but standing crop was high. Similarly, within each year the greatest dietary overlap (Fig. 3) corresponded to lowest dietary quality (Fig. 2) and highest plant biomass availability (Fig. 4). Also, between years, dietary overlap was greater when plant biomass was greatest (Fig. 4) in the spring-summer of 1981 relative to 1980 (Fig. 3). In summary, dietary overlap between elk and deer at Tomales Point is greatest, i.e., competitive interactions are relaxed, during periods of abundant low quality forage, and overlap is least, $i$. e., competitive interactions are intensified, during periods of less abundant, high quality forage. Thus, our results support the hypothesis of lower dietary 


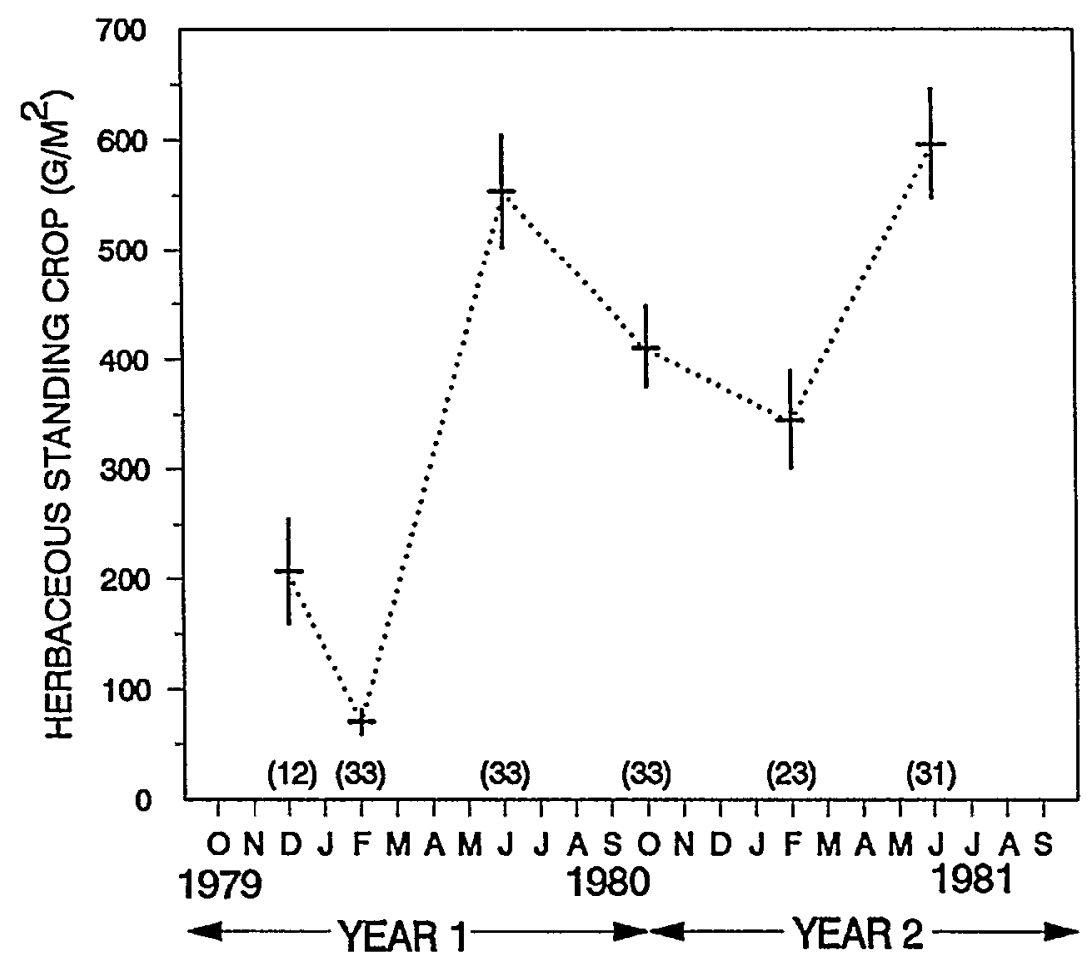

Fig. 4. Mean and standard error (vertical bar) of herbaceous standing crop within the grassland type at Tomales Point from December 1979 through June 1981. Parentheses enclose sample size for each sample period.

overlap during periods of limited forage availability (Hobbs et al. 1983, Schoener 1986) and are not consistent with the hypothesis of lower dietary overlap in periods of forage abundance (Nudds 1980, Westoby 1974).

Our findings for tule elk and black-tailed deer in a prairie-scrub mosaic in coastal California contrast to the results of a parallel study of Roosevelt elk and black-tailed deer in the old-growth forest of the Olympic Peninsula, Wash., where dietary overlap was greatest in the winter months (Leslie et al. 1984), coincident with low forage quantity (Leslie 1983) and quality (Leslie and Starkey 1985). However, in both our California study and in Washington (Leslie et al. 1984, Leslie and Starkey 1985), dietary overlap was greatest when fecal nitrogen was lowest, suggesting that forage quality rather than forage abundance alone influences levels of dietary overlap. This study raises this issue rather than resolves it.

Levels of dietary overlap between tule elk and black-tailed deer in summer and fall of both years at Tomales Point were comparable to those for Roosevelt elk and deer on the Olympic Peninsula (Leslie et al. 1984) in spite of the low elk density at Tomales Point during this study. Undoubtedly, range conditions induced by high cattle stocking rates and a drought in 1978-79 influenced forage availability throughout the life of this study. Nevertheless, levels of dietary overlap and dietary quality were quite similar in 1980 and 1981 in spite of increased amounts of available forage in 1981. The tule elk suffered from copper deficiency in 1979, but this had been alleviated by 1980 (Gogan et al. 1989). Numbers of tule elk at Tomales Point failed to increase during this study, but subsequently increased at a rate approximating the maximum potential from 1981 to 1984 (Gogan and Barrett 1986). They have continued increasing from 1984 to 1994 (W. Shook, pers. commun.). Deer densities have not been determined since 1981.

\section{Literature Cited}

Abrams, P. 1980. Some comments on measuring niche overlap. Ecol. $61: 44-49$.

Anderson, A. E., and O. C. Wallmo. 1984. Odocoileus hemionus. Mammalian Species 219:1-9.

Anthony, R. G., and N. S. Smith. 1974. Comparison of rumen and fecal analysis to describe deer diets. J. Wildl. Manage. 38:535-540.

Anthony, R. G., and N. S. Smith. 1977. Ecological relationships between mule deer and white-tailed deer in southeastern Arizona. Ecol. Monogr. 47:255-277.

Azevedo, J., and D. L. Morgan. 1974. Fog precipitation in coastal California forests. Ecol. 55:1135-1141.

Barbour, M. G., R. B. Craig, F. R. Drysdale, and M. T. Shiselin. 1973. Coastal ecology: Bodega Head. Univ. Califomia Press, Berkeley, Calif.

Clark, J. 1986. Tule elk-back from the brink. Outdoor California 46:2-11.

Dougherty, S., W. Faris, and L. Nitikman. 1978. A vegetational survey of tule elk rangeland, Point Reyes National Seashore. Environmental Studies. Univ. California, Santa Cruz, Calif.

Ellis, J. E., J. A. Wiens, C. F. Rodell, and J. C. Anways. 1976. A conceptual model of diet selection as an ecosystem process. J. Theor. Biol. 60:93-108.

Elliott, H. W., III, and R. II. Barrett. 1985. Dietary overlap among axis, fallow and black-tailed deer and cattle. J. Range Manage. 38:435-439.

Evans, R. A., and M. Love. 1957. The step-point method of sampling-a practical tool in range research. J. Range Manage. 10:208-212.

Fowler, G. S. 1985. Tule elk in California: history, current status, and management recommendations. Dep. of Forest. and Resource Manage. Univ. California, Berkeley, Calif.

Gogan, P. J. P. 1986. Ecology of the Tule Elk Range, Point Reyes National Seashore. Ph.D. Thesis, Univ. Califomia, Berkeley, Calif.

Gogan, P. J. P., and R. H. Barrett. 1986. Comparative dynamics of two reintroduced elk herds. J. Wildl. Manage. 51:20-27. 
Gogan, P. J. P., and R. H. Barrett. 1994. Roosevelt elk dietary quality in northern coastal California. Calif. Fish Game 80:80-\$3.

Gogan, P. J. P., D. A. Jessup, and M. Akeson. 1989. Copper deficiency in tule elk at Point Reyes. California. J. Range Manage. 42:233-238.

Gogan, P. J. P., D. A. Jessup, and R. H. Barrett. 1988. Antler anomalies in tule elk. J. Wild!. Dis. 24:656-662.

Hansen, R. M., T. M. Foppe, M. B. Gilbert, R. C. Clark, and H. W. Reynolds. 1977. The microhistological analysis of feces as an estimator of herbivore diet. Colorado State Univ. Composition Analaysis Laboratory, Fort Collins, Colo.

Harper, J. A., J. H. Harn, W. W. Bentley, and C. F. Yocum. 1967. The status and ecology of Roosevelt elk in California. Wildl. Monogr. 16.

Heady, H. F., T. C. Foin, M. K. Hektner, D. W. Taylor, M. S. Barbour, and W. J. Barry. 1977. Coastal prairie and northern coastal scrub, p. 733-757. In: M. S. Barbour and J. Major (eds.), Terrestrial vegetation of California. John Wiley and Sons, Inc., N. Y.

Hobbs, N. T. 1987. Fecal indices to dietary quality: a critique. J. Wildl. Manage. 51:317-320.

Hobbs, N. T., D. L. Baker, and R. B. Gill. 1983. Comparative nutritional ecology of montane ungulates during winter. J. Wildl. Manage. 47:1-16.

Hobbs, N. T., D. L. Baker, J. E. Ellis, and D. M. Swift. 1981. Composition and quality of elk winter diets in Colorado. J. Wild. Manage. 45:156-171.

Holechek, J. L., M. Vavra, and R. D. Pieper. 1982. Botanical composition determination of range herbivore diets: a review. J. Range Manage. 35:309-315.

Horowitz, W. 1980. Official methods of analysis of the Association of Official Analytical Chemists. Thirteenth ed. Assoc. Off. Anal. Chem., Washington, D.C.

Howell, J. T. 1970. Marin flora. 2nd ed. Univ. California Press, Berkeley, Calif.

Hurlbert, S. H. 1978. The measurement of niche overlap and some relatives. Ecol. 59:67-77.

Kie, J. G., D. L. Drawe, and G. Scott. 1980. Changes in diet and nutrition with increased herd size in Texas white-tailed deer. J. Range. Manage. 33:28-34.

Kie, J. G., and T. S. Burton. 1984. Dietary quality, fecal nitrogen and 2, 6 diaminopimelic acid in black-tailed deer in northern California. Res. Note PSW-36t. Berkeley, Calif.

Kuchler, A. W. 1977. The map of the natural vegetation of California, p. 909-938. In: M. G. Barbour and J. Major (eds), Terrestrial vegetation of Califomia. John Wiley and Sons, Inc., N. Y.

Lathrop, K. T., and P. J. P. Gogan. 1985. Plant communities of the tule elk range Point Reyes National Seashore. Cooperative National Park Resources Studies Unit, Univ. of California, Davis, Calif. Tech. Rep. 18.

Leslie, D. M., Jr. 1983. Nutritional ecology of cervids in old-growth forests in Olympic National Park, Washington. Ph.D. Diss., Oregon State Univ., Corvallis, Ore.

Leslie, D. M., Jr., and E. E. Starkey. 1985. Fecal indices to dietary quality of cervids in old-growth forests. J. Wildl. Manage, 49:142-146.

Leslie, D. M., Jr., and E. E. Starkey. 1987. Fecal indices to dietary quality: a reply. J. Wildl. Manage. 51:321-325.

Leslie, D. M., Jr., E. E. Starkey, and M. Varra. 1984. Elk and deer diets in old-growth forests in western Washington. J. Wildl. Manage. 48:762-775.

Leslie, D. M., Jr., M. Vavra, E. E. Starkey, and R. C. Slater. 1983. Correcting for differential digestibility in microhistological analyses involving common coastal forages of the Pacific northwest. J. Range. Manage. 36:730-732.

Longhurst, W. M., A. S. Leopold, and R. F. Dasmann. 1952. A survey of California deer herds, their range and management problems. Dep. Fish and Game, Sacramento, Calif. Game Bull. No. 6.

Longhurst, W. M., E. O. Garton, H. F. Heady, and G. E. Connolly. 1976. The California deer decline and possibilities for restoration. West. Sect. Wild. Soc. 1976:74-103

Longhurst, W. M., G. E. Connolly, B. M. Browning, and E. O. Garton. 1979. Food interrelationships of decr and sheep in parts of Mendocino and Luke Counties, Calif. Hilgardia 47:191-247.

Martin, T. E. 1993. Nest predation and nest sites. BioScienee 43:523-532. McCullough. D. R. 1969. The tule elk: its history, behavior and ecology. Univ. California Publ. Zool. 88 .

Nelson, J. R., and T. A. Leege. 1982. Nutritional requirements and food habits, p. 323-367. In: J. E. Thomas and D. E. Toweill (eds), Elk of North America: ecology and management. Stackpole Books, Harrisburg, Penn.
Nudds, T. D. 1980. Forage "preference": theoretical considerations of diet selection by deer. J. Wildl. Manage. 44:735-740.

Phillips, J. A. 1985. Acclimation of reintroduced tule elk in the Diablo Range, California. M.S. Thesis. San Jose State Univ., San Jose, Calif.

Putman, R. J. 1986. Competition and coexistence on a multi-species grazing system. Acta Theriol. 31:271-291.

Ray, D. T. 1981. Post-release activity of tule at Point Reyes National Scashore, California. M. S. thesis, Univ. of Michigan, Ann Arbor.

Schoener, T. W. 1970. Non-synchronous spatial overlap of lizards in patchy habitats. Ecol. 51:408-418.

Schoener, T. W. 1986. Resource partitioning, p. 91-126. In: D. Anderson and J. Kikkawa (eds), Community Ecology-Pattern and Process. Blackwell, Oxford, U. K.

Sparks, D. R., and J. C. Malechek. 1968. Estimating percentage dry weight in diets using a microscopic technique. J. Range Manage. 21:26+-265.

Strong, D. R., Jr. 1983. Natural variability and manifold mechanisms of ecological communities. Amer. Natur. 122:636-660. Strauss, D., and D. L. Neal. 1983. Biases in the step-point method on bunchgrass ranges. J. Range Manage. 36:623-626.

Strauss, R. E. 1979. Reliability estimates for Ivlev's electivity index, the foraging ratio, and a proposed linear index of forage selection. Trans. Am. Fish. Soc. 108:344-352.

Taber, R. D., and R. F. Dasmann. 1958. The black-tailed deer of the chaparral: its life history and management in the North Coast Range of California. Dep. Fish and Game, Sacramento, Calif. Game Bull. No. 8.

Todd, J. W., and E. M. Hansen. 1973. Plant fragments in the feces of bighorns as indicators of food habits. J. Wildl. Manage. 37:363-366.

Truman, C. F., J. Dushek, and R. H. Barrett. 1983. A staining procedure for plant epidermal fragments, p. 146-150 $m$ : H. W. Elliott. III, Ecological relationships of cattle, axis deer, fallow deer, and black-tailed deer on Point Reyes Peninsula. Ph.D. Thesis, Univ. Califormia, Davis.

U.S. Dep. of Commerce, Weather Bureau. 1942. General Summary. Climatological data, Califi. Section 26:145-156.

Vavra, M., and J. L. Holechek. 1980. Factors influencing microhistological analysis of herbivore diets. J. Range Manage. 33:371-374.

Westoby, M. 1974. An analysis of diet selection by large generalist herbivores. Amer. Natur. 108:290-304.

Wiens, J. A. 1979. On competition and variable environnnents. Amer. Sci. 65:590-597. 